\title{
Flow and heat transfer characteristics of tornado-like vortex flow
}

\author{
Y. Suzuki \& T. Inoue \\ Department of Mechanical Sciences and Engineering, \\ Tokyo Institute of Technology
}

\section{Abstract}

A tornado-like vortex flow has been investigated for both effective local ventilation from the floor of a room and local heat transfer control without the circumferential thermal diffusion. A relatively stable axisymmetrical tornadolike vortex is generated in a water tank by a vertically uniform swirl. The overall flow structure of the tornado-like vortex has been categorized into three regions: the outer free vortex region, the inflow layer (the Ekman boundary layer), and the vortex tube. The present tornado-like vortex flow was investigated experimentally and also agrees qualitatively with this flow structure. In the free vortex region, the tangential velocity distribution shows a Rankine-like vortex and has good agreement with an approximate formula characterized by the radius and the maximum tangential velocity of the forced vortex. A numerical analysis using an axisymmetrical laminar flow model is carried out. To eliminate the effect of swirl supplying methods, a virtual cylindrical surface of the radius $\left(R_{\text {out }}\right)$ is considered, where an axisymmetrical flow is established. All experimental and computational data of the flow characteristics of the tangential velocity profile in the free vortex region are well-arranged by the Reynolds number based on the flow rate and the swirl Reynolds number based on $R_{\text {out }}$. The flow structure in the Ekman boundary layer is characterized by the tangential velocity profile in the free vortex region and the height where the radial velocity is half its maximum value. The heat transfer characteristics of the heated plate with uniform heat flux located on the bottom are investigated both experimentally and computationally. The forced convective heat transfer of the tornado-like swirling flow is handled in the same way as the laminar forced convective heat transfer on a flat plate, by using the maximum radial velocity component in the Ekman boundary layer.

Keywords: tornado-like vortex, swirl flow, flow structure, heat transfer control. 


\section{Introduction}

The tornado is an intensive forced columnar vortex with a strong updraft above a lower boundary. For a better understanding of the dynamics of naturally occurring intense vortices such as tornadoes, there are a number of approaches from both meteorological and mechanical points of view [1]. A tornado-like swirling flow has been more prospective for effective local ventilating from the room floor where polluted air and dust arise, and for local heat transfer control on the base without the circumferential thermal diffusion. We have investigated the local ventilation and heat transfer characteristics of the tornado-like swirling flow by using the top rotating vortex generator [2]. However, the velocity field and heat transfer characteristics of the tornado-like vortex have not been clarified enough for handling of such a system.

The objective of the present work is as follows: to clarify the flow structure of the tornado-like swirling flow generated by a vertically uniform swirl flow from the peripheral surface and a suction from the center top, and to investigate the heat transfer on the heated bottom plate.
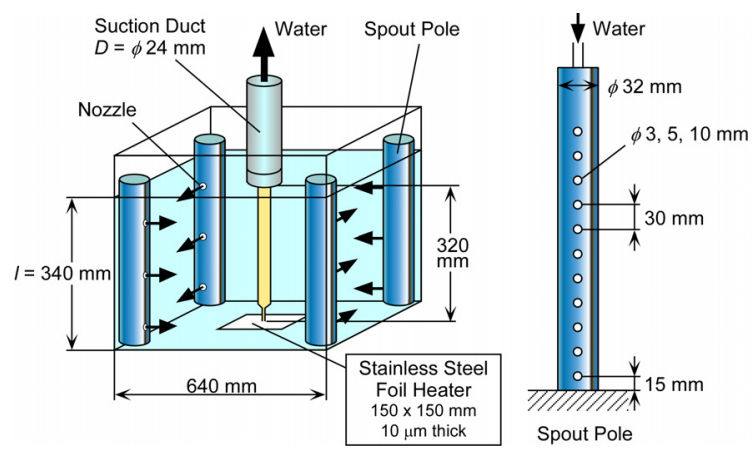

Figure 1: $\quad$ Experimental setup.

\section{Experimental apparatus and procedures}

The experimental apparatus is shown in Fig. 1. A tornado-like vortex flow is generated in a square water tank made of acrylic resin, which size is $640 \mathrm{~mm}$ square. The water level is fixed in height $l=340 \mathrm{~mm}$. A relatively-stable axisymmetrical tornado-like vortex is generated by a vertically-uniform swirl from four cornered poles with water nozzles and the suction from the duct (inner diameter, $D=24 \mathrm{~mm}$ ) installed at a height of $320 \mathrm{~mm}$ from the base in the center of the tank. The suction and supply flow rate $(Q)$ is varied between 1.0 to 5.0 $l /$ min. The swirl intensity is varied by the diameter of the water nozzle $(d=3,5$, $10 \mathrm{~mm})$. The supply water is controlled at a constant temperature without the influence of the bulk temperature change. The velocity components of the tornado-like vortex flow are measured by PTV. To obtain the detail of velocity field of the vortex boundary layer near the base, the radial and tangential velocity components are measured by 2D-LDV put under the base plate. 
The same experimental apparatus is used to obtain the local heat transfer coefficient through knowledge of the fixed surface heat flux, the bulk temperature, and the local wall temperature. For measuring the local wall temperature, 57 T-type thermocouples are installed on the backside of the stainless steel foil heater $(150 \mathrm{~mm}$ square, $10 \mu \mathrm{m}$ thick) radially from the center of the tank. The fixed surface heat flux is varied between $1.7 \times 10^{2}$ and $2.7 \times 10^{3} \mathrm{~W} / \mathrm{m}^{2}$.

\section{Results and discussion}

\subsection{Flow structure of tornado-like swirling flow}

\subsubsection{Overall flow structure}

The present tornado-like vortex flow is visualized as shown in Fig. 2. The dye put on the base is concentrated at the center of the vortex, lifted up and evacuated from the suction duct. The vortex breakdown is shown near the base. An overall flow structure of the tornado-like vortex has been categorized into three regions [1]: a center vortex tube with a spiral upward flow, the outer free vortex region with a tangential velocity profile of a Rankine-like vortex, and an inflow layer near the base called as the Ekman boundary layer. The present tornado-like swirling flow also agrees qualitatively with this flow structure as shown in Fig.3.

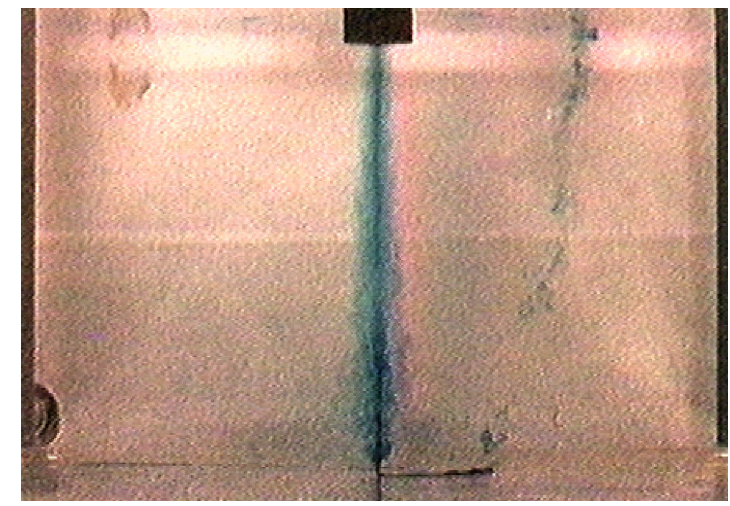

Figure 2: Visualization of tornado-like vortex flow: $Q=5.0 \mathrm{l} / \mathrm{min}, d=3 \mathrm{~mm}$.

\subsubsection{Tangential velocity distribution in free vortex region}

In the free vortex region, which comprises a large part of a tornado-like swirling flow, the tangential velocity distribution shows a Rankine-like vortex. The tangential velocity distributions are almost same at any height except near the suction duct and in the Ekman boundary layer. The larger flow rate and swirl intensity provide the higher maximum velocity $\left(v_{\theta \max }\right)$ and the smaller radius of the forced vortex $\left(R_{F}\right)$.

In the present experimental apparatus, it is very difficult to estimate the tangential velocity component in the outer flow region, $v_{\theta \text { out }}$, from the initial velocity of the water nozzles as the given boundary condition. To eliminate the 
effect of swirl supplying methods, a virtual peripheral cylindrical surface of the radius $R_{\text {out }}$ is considered, where an axisymmetrical flow is established. In this case, $R_{\text {out }}=200 \mathrm{~mm}$. An approximate expression of the tangential velocity distribution in the free vortex region is considered as Eq. (1), which superimposes the effect of the outer given swirling flow on a Rankine-like vortex flow.

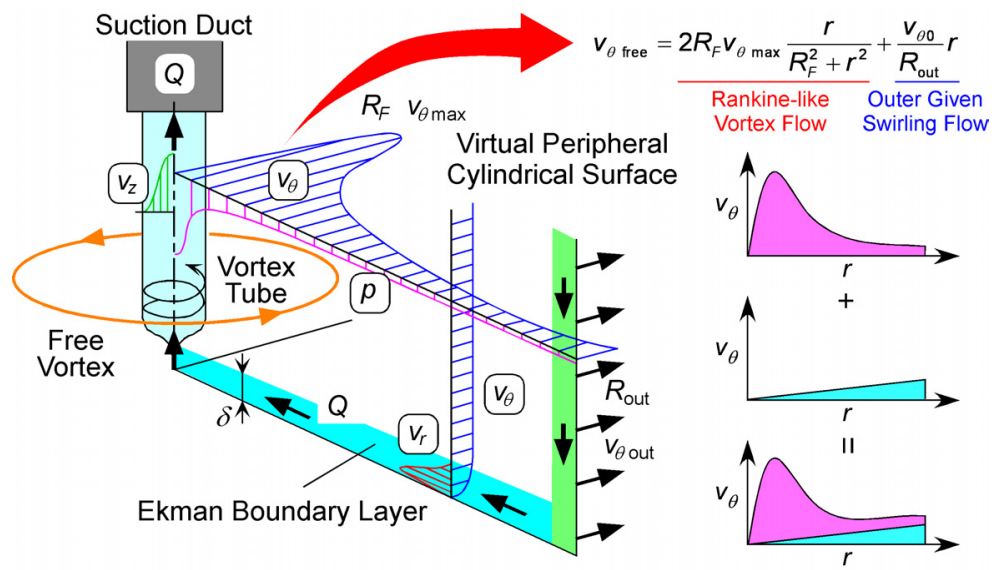

Figure 3: $\quad$ Structure of tornado-like vortex flow.

$$
v_{\theta \text { free }}=2 R_{F} v_{\theta \max } \frac{r}{R_{F}^{2}+r^{2}}+\frac{v_{\theta 0}}{R_{\text {out }}} r
$$

The tangential velocity distribution has good agreement with Eq. (1), which is characterized by the radius and the maximum tangential velocity of the forced vortex $\left(R_{F}\right.$ and $\left.v_{\theta \max }\right)$. The tangential velocity at virtual cylindrical surface $\left(v_{\theta \text { out }}\right)$ is obtained by setting $r=R_{\text {out }}$ in Eq. (1).

\subsubsection{Numerical simulation}

A numerical analysis using an axisymmetrical laminar flow model is carried out to investigate the structure of the tornado-like vortex flow. The calculation domain is $14 D \times 12 D$ in the $z$ and $r$ directions on the basis of the suction duct diameter $D$. The calculation grids are set by $320 \times 280$ with non-uniform grid spacing near the center and the wall regions. The inflow boundary conditions at $r=12 D$ are uniform velocities of the radial and tangential components $\left(v_{r}\right.$ and $\left.v_{\theta}\right)$ respectively. The radius of the virtual cylindrical surface corresponds to $R_{\text {out }}=$ $8.33 D$. As the dominant parameters for the flow field, the Reynolds number $R e$ based on the flow rate and the swirl Reynolds number $R e_{s}$ based on the circulation at the virtual surface are defined as $R e=(Q / l) / v, R_{s}=\left(R_{\text {out }} v_{\theta \text { out }}\right) / v$.

The dimensionless form of Eq. (1) is given by using the radius and the velocity of the virtual cylindrical surface $\left(R_{\text {out }}\right.$ and $\left.v_{\theta \text { out }}\right)$ as follows.

$$
v_{\theta \text { free }}^{*}=2 R_{F}^{*} v_{\theta \max }^{*} \frac{r^{*}}{R_{F}^{* 2}+r^{* 2}}+C^{*} r^{*}
$$


The computational results for the tangential velocity profiles in the free vortex region have good agreement with Eq. (2) as well as experimental results. Both experimental and computational flow characteristics of the tangential velocity profile in the free vortex region of this swirling flow are well-arranged by $R e$ and $R e_{s}$ as shown in Fig. 4. These flow characteristics are estimated as follows:

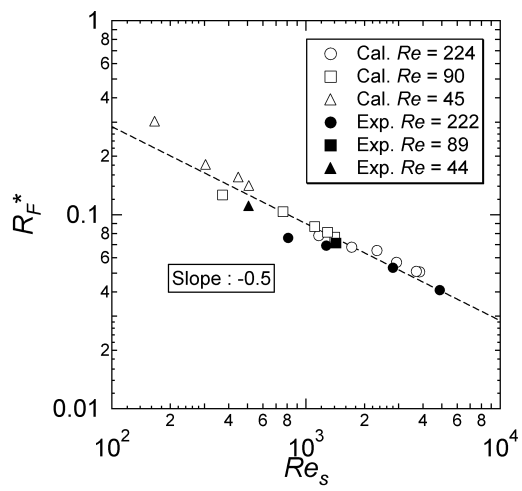

(a) Radius of forced vortex, $R_{F}{ }^{*}$

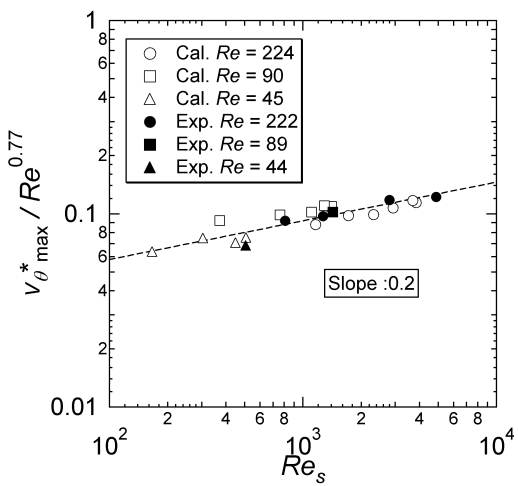

(b) Maximum tangential velocity, $v_{\theta \max }^{*}$

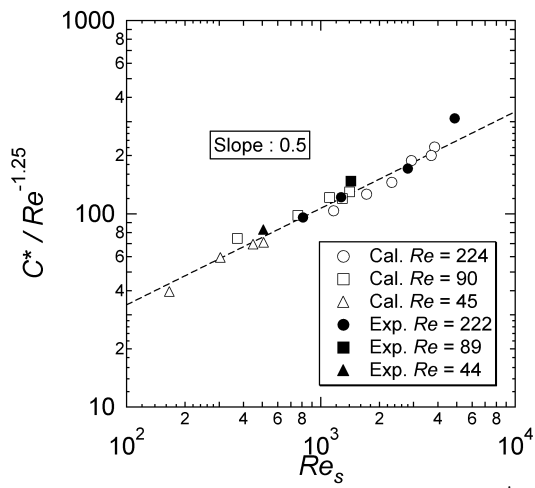

(c) Tangential velocity ratio, $C^{*}$

Figure 4: Flow characteristics in free vortex region.

$$
\left.\begin{array}{l}
R_{F}^{*} \equiv \frac{R_{F}}{R_{\text {out }}} \approx 2.84 \operatorname{Re}_{s}^{-0.5} \\
v_{\theta \max }^{*} \equiv \frac{v_{\theta \max }}{v_{\theta \text { out }}} \approx 0.0231 \operatorname{Re}^{0.77} R e_{s}^{0.2} \\
C^{*} \equiv \frac{v_{\theta 0}}{v_{\theta \text { out }}} \approx 3.38 R e^{-1.25} \operatorname{Re}_{s}^{0.5}
\end{array}\right\},
$$

where the constant numbers are considered as dependent values on geometric parameters such as an aspect ratio which is constant in the present work. The radius of the forced vortex $R_{F}^{*}$ is not dependent on $R e$, that is the total flow rate, but in proportion to $\operatorname{Re}_{s}^{-0.5}$. This result is consistent with Khoo et al. [3]. 
Meanwhile, the maximum tangential velocity $v_{\theta \max }{ }^{*}$ is rather influenced by $R e$. Here $v_{\theta 0}$ means the tangential velocity of the totally forced vortex that is generated by the outer swirling flow without the base plate. Thus, $1-C^{*}$ is considered as the ratio of the angular momentum transported from the outer flow region to the vortex center through the Ekman boundary layer. The larger flow rate and the lower swirl intensity provide the higher transport ratio.

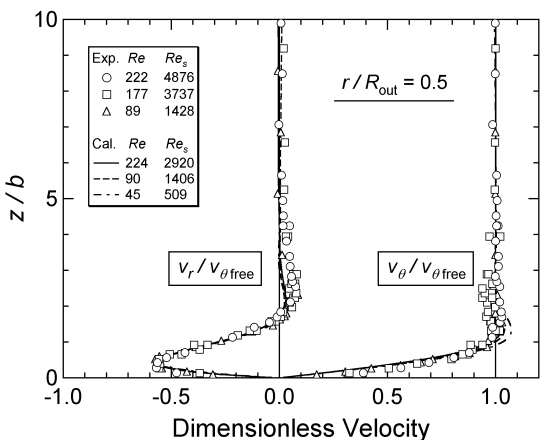

(a) $r^{*}=r / R_{\text {out }}=0.5$

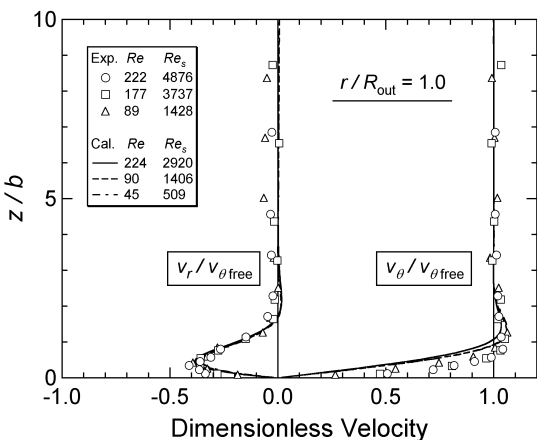

(b) $r^{*}=1.0$

Figure 5: Dimensionless velocity profile in Ekman boundary layer.

\subsubsection{Flow structure in Ekman boundary layer}

The velocity distributions of the Ekman boundary layer is investigated both experimentally and computationally. Figure 5 shows the radial and tangential velocity profiles by using $2 \mathrm{D}-\mathrm{LDV}$ and calculating. The normalization by the tangential velocity of the free vortex region $\left(v_{\theta \text { free }}\right)$ and the height $(b)$ where $\left|v_{r}\right|=$ $v_{r \text { max }} / 2$ brings the consistent velocity profiles at each radius. Furthermore, the radial profiles of the maximum radial velocity component and the characteristic height $b$ are shown in Fig. 6 and estimated as follows:

$$
\left.\begin{array}{l}
\frac{v_{r \max }}{v_{\theta \text { free }}} \approx-0.46 r^{*}+0.83 \\
b^{*} \equiv \frac{b}{R_{\text {out }}} \approx\left(2.28-0.60 \exp \left(-6.21 r^{* 2}\right)\right) R e^{0.23} R e_{s}^{-0.65}
\end{array}\right\},
$$

except near the vortex center where the upward flow has large influence. The maximum radial velocity is increasing linearly as the radius is decreasing. The radial profile of the Ekman boundary layer thickness is estimated as 1.7b. This estimation has good agreement with results of the boundary layer visualization. The Ekman boundary layer thickness is decreasing as the radius is decreasing.

\subsection{Effect of tornado-like vortex on heat transfer from heated bottom plate}

\subsubsection{Local heat transfer coefficient}

Additionally, the heat transfer characteristics of the heated plate with uniform heat flux located on the bottom surface of the tornado-like vortex are investigated experimentally. Since the value of the fixed surface heat flux has no 
difference in the heat transfer characteristics, the results of the maximum heat flux $\left(q=2.7 \times 10^{3} \mathrm{~W} / \mathrm{m}^{2}\right)$ in this work are discussed. This means that the forced convection of the tornado-like vortex flow is more dominant than the natural convection. As the swirl intensity is higher, the higher temperature region concentrates near the heater center, and the overall temperature on the heater surface goes down. The temperature distribution has rotational symmetries through 90 degrees. On the diagonal line of the heater, the relatively higher temperature region is confirmed. Thus, the profile of the local heat transfer coefficient $\left(h=q /\left(T_{w}-T_{b}\right)\right)$ on the transversal lines crossing the center and the diagonal lines of the heater surface are examined as shown in Fig. 7. The effect of the heat concentration to the center brings the decreasing of the heat transfer coefficient near the vortex center, but the overall improvement is occurred with increasing of the swirl intensity. The flow quite near the base goes approximately straight toward the center. The heat transfer characteristics on the square heater have the path dependency, and are concerned with between the heater shape and the radial velocity component in the Ekman boundary layer.

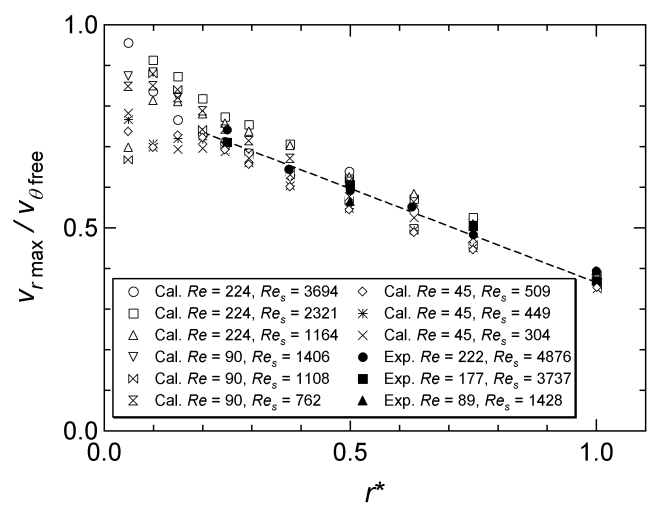

(a) Dimensionless maximum radial velocity profile, $v_{r \text { max }} / v_{\theta \text { free }}$

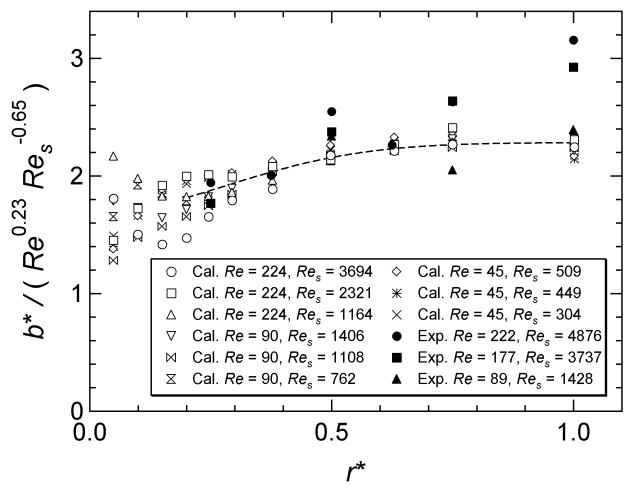

(b) Dimensionless characteristics scale profile, $b^{*}$

Figure 6: $\quad$ Flow characteristics in Ekman boundary layer. 


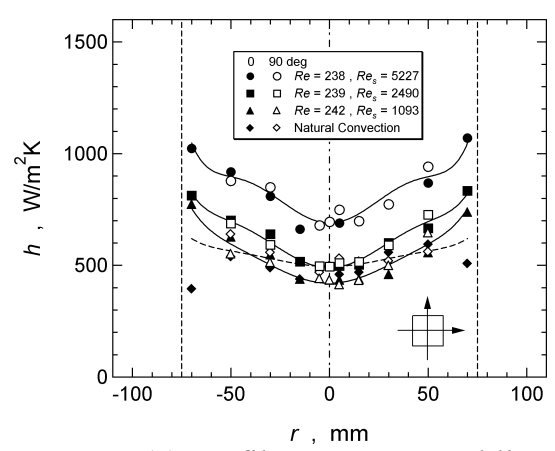

(a) Profile on transversal lines

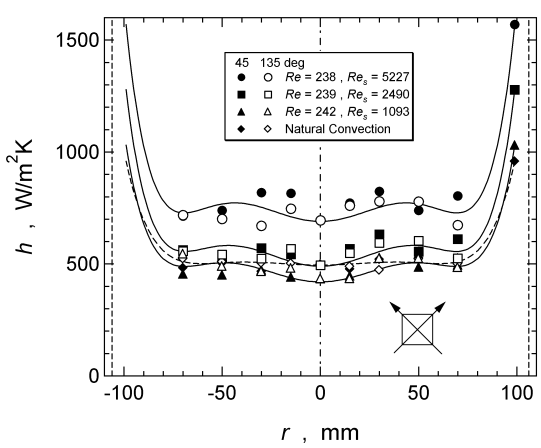

(b) Profile on diagonal lines

Figure 7: Heat transfer coefficients on heated bottom plate.

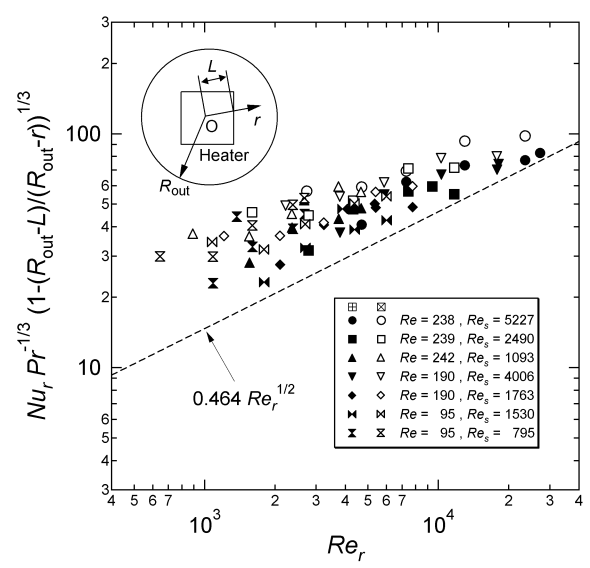

Figure 8: $\quad$ Correlation between $N u_{r}$ and $R e_{r}$ on heated bottom plate.

\subsubsection{Local Nusselt number and local Reynolds number}

The forced convective heat transfer characteristics on the line from the virtual peripheral cylindrical surface to the heater center through the unheated section and the uniform heat flux section are investigated. The local Nusselt number and the local Reynolds number are defined as follows: $N u_{r}=h\left(R_{\text {out }}-r\right) / \lambda$ and $R e_{r}=$ $v_{r \max }\left(R_{\text {out }}-r\right) / v$, where $v_{r \max }$ is the maximum radial velocity component in the Ekman boundary layer, to which the above Eqs. (2) and (3) in the flow characteristics of the tornado-like vortex flow are applied. Figure 8 shows the correlation between $N u_{r}$ and $R e_{r}$ referred to an unheated starting length problem of a laminar forced convective heat transfer on a flat plane, where $L$ means the slant distance from the center to the edge of the heater. In Fig. 8, the data on both the axes and the diagonal are distributed on an almost same straight line. The local Nusselt number is rather large than a laminar forced convective heat transfer on a flat plane with a uniform heat flux through an unheated starting length shown as Eq. (5) [4], but it demonstrates a qualitatively almost same 
tendency without reference to the flow rate $(R e)$ and the swirl intensity $\left(R e_{s}\right)$ of the tornado-like vortex flow.

$$
N u_{r}=0.464 P r^{1 / 3} \operatorname{Re}_{r}^{1 / 2}\left(1-\frac{R_{\text {out }}-L}{R_{\text {out }}-r}\right)^{-1 / 3}
$$

\subsubsection{Effect of free convection from heated bottom plate}

The forced convective heat transfer is dominant above the surface of the heated bottom plate. The effect of free convection from the heated bottom plate is investigated computationally. A numerical analysis using a same axisymmetrical laminar flow model above the circular heater with the uniform heat flux is carried out. The radius of the circular heater $\left(L_{m}\right)$ is set as same surface area as the experiments. The modified Grashof number is defined as $G r^{*}=g \beta q L_{m}{ }^{4} / v^{2} \lambda$. In case of $\mathrm{Gr}^{*} \leq 7.0 \times 10^{6}$, flow characteristics are almost never affected by the free convection. In case of $\mathrm{Gr}^{*}>7.0 \times 10^{6}$, as the heat flux is increasing, the flow concentrates in the vortex center in the Ekman boundary layer. The Ekman boundary layer thickness near the center is thinner as shown in Fig. 9(a). Furthermore, the mean Nusselt number $\left(N u_{m}=h L_{m} / \lambda\right)$ near the center is increasing as shown in Fig. 9(b). However, in almost all area except the center of vortex, the free convection has no influence upon the flow and heat transfer characteristics.

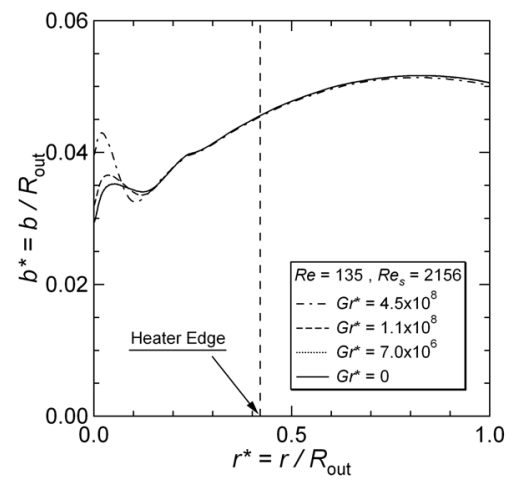

(a) Ekman boundary layer thickness

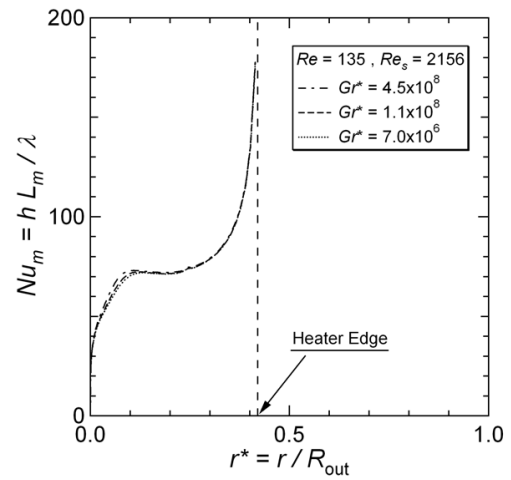

(b) Mean Nusselt Number

Figure 9: Effect of free convection on heated bottom plate.

The correlations between the local Nusselt number $\left(N u_{L-r}=h(L-r) / \lambda\right)$ and the local Reynolds number $\left(\operatorname{Re}_{L-r}=v_{r \max }(L-r) / v\right)$ based on the distance from the heater edge $(L-r)$, are shown in Fig. 10. In the case that the forced convective heat transfer is dominant (Fig. 10(b)), both experimental and computational results have good agreement with $N u_{L-r} \propto R e_{L-r}^{0.5}$ without the influence of $\mathrm{Gr}^{*}$. On the other hand, the upward flow depend on the free convection brings the lower heat transfer near the center of vortex (Fig. 10(a)). 


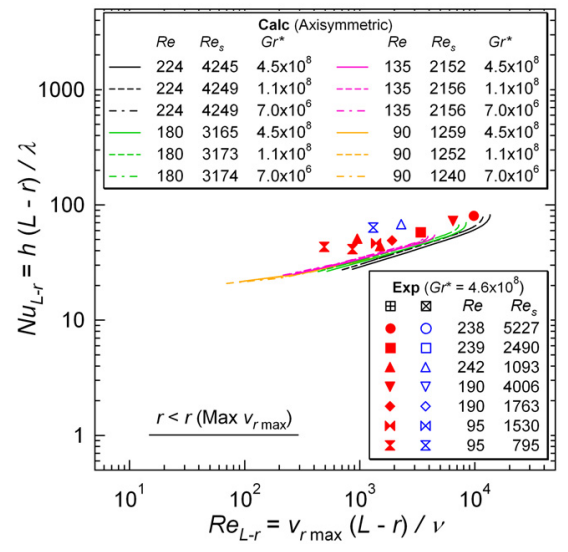

(a) $r<r\left(\operatorname{Max} v_{r \max }\right)$

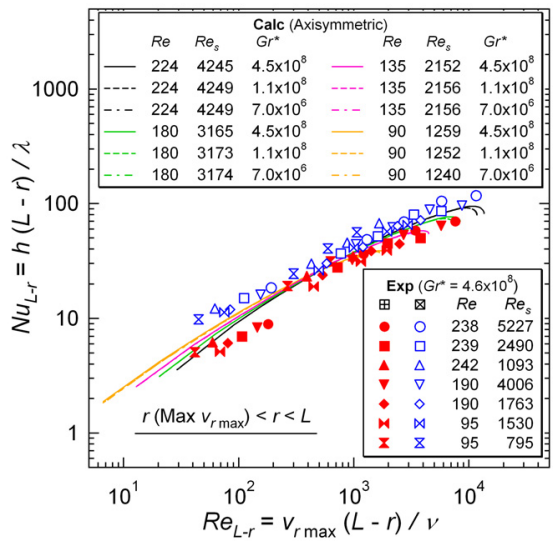

(b) $r\left(\operatorname{Max} v_{r \max }\right)<r<L$

Figure 10: $\quad$ Correlation between $N u_{L-r}$ and $R e_{L-r}$ on heated bottom plate.

\section{Conclusions}

(1) In the free vortex region of the tornado-like swirling flow, the forced vortex radius, the maximum tangential velocity, and the tangential velocity ratio is in proportion to $R e_{s}^{-0.5}, \operatorname{Re}^{0.77} R e_{s}^{0.2}$, and $R e^{-1.25} R e_{s}^{0.5}$ respectively.

(2) The flow structure in the Ekman boundary layer is characterized by the tangential velocity profile described as above in the free vortex region, and the characteristic scale $b$ defined as the height where the radial velocity is half its maximum value. The Ekman boundary layer thickness is estimated as $1.7 b$.

(3) On the base of the tornado-like swirling flow, the forced convective heat transfer is dominant except the center of vortex, and handled as same as the laminar forced convective heat transfer on a flat plate, by using the maximum radial velocity component in the Ekman boundary layer.

\section{References}

[1] Snow, J.T., A Review of Recent Advances in Tornado Vortex Dynamics. Reviews of Geophysics and Space Physics, 20(4), pp. 953-964, 1982.

[2] Hijikata, K., Suzuki, Y., Aizawa, Y. \& Kozawa, Y., Local Ventilation by Tornadolike Vortex. Transactions of the Japan Society of Mechanical Engineers (in Japanese), 61(587) B, pp. 401-407, 1995.

[3] Khoo, B.C., Yeo, K.S. \& Yao, S.E., Effusing Core at the Center of a Potential Vortex. Experimental Thermal and Fluid Science, 7, pp. 307318, 1993.

[4] The Japan Society of Mechanical Engineers, JSME Mechanical Engineers' Handbook, A6: Thermal Engineering (in Japanese), Maruzen: Tokyo, 1985. 